\title{
De la presencialidad a la virtualidad ante la pandemia de la Covid-19: impacto en docentes universitarios
}

\author{
Carlos L. Chanto Espinoza*1; Marcela Mora Peralta ${ }^{2}$ \\ ${ }^{1}$ http://orcid.org/0000-0002-3420-7259, ${ }^{2}$ http://orcid.org/0000-0003-0115-7395, ${ }^{1,2}$ Universidad Nacional, Guanacaste, Costa Rica
}

Citar como: Chanto, C., \& Peralta, M. (2021). De la presencialidad a la virtualidad ante la pandemia de la Covid-19: impacto en docentes universitarios. Revista Digital de Investigación en Docencia Universitaria, 15(2), e1342. https://doi. org/10.19083/10.19083/ridu.2021.1342

Recibido: 28/09/2020. Revisado: 04/11/2020. Aceptado: 01/09/2021. Publicado: 26/11/2021.

\begin{abstract}
Resumen
Introducción: En el marco de la pandemia provocada por la COVID-19, cada docente asumió el reto de reinventarse rápidamente y empezar a trabajar de otra forma, diseñando actividades de aprendizaje y evaluativas en la presencialidad remota. Objetivo: establecer el impacto del cambio metodológico del proceso de enseñanza-aprendizaje, de modalidad presencial a modalidad virtual, en los académicos de la Universidad Nacional de Costa Rica - Campus Liberia. Método: En el estudio participaron 85 personas Docentes de las diferentes carreras del centro universitario, bajo un muestreo no probabilístico. Se empleó un cuestionario estructurado y cerrado de escasas preguntas enviado por correos electrónicos institucionales y por la vía telefónica mediante la aplicación digital WhatsApp. Resultados: La mejora de la mediación pedagógica tiene estrecha relación con el acceso a la tecnología, tanto el equipo como el acceso al internet, y esta ha sido considerada una limitante para que el personal académico pueda comunicarse de manera fluida con la población estudiantil. Discusión: La academia debe tener claridad sobre las condiciones de la población estudiantil, la cual no tiene las facilidades de acceso a las herramientas tecnológicas, conectividad y, especialmente, condiciones económicas, sociales y afectivas.
\end{abstract}

Palabras clave: docencia; aprendizaje en línea; educación; aprendizaje; COVID-19.

\section{From Face-to-Face to Virtual Mode Because of Covid-19: Impact in the Teaching-Learning Process}

\begin{abstract}
Introduction: Within the framework of the pandemic provoked by the COVID-19, every teacher took the challenge to rebrand quickly and start working differently by designing and learning evaluative activities to face this E-Learning environment in which most students and teachers all around the world have been immersed for so long. Objective: To establish the impact of the methodological change within the traditional and virtual classroom in academics of Universidad Nacional of Costa Rica - Campus Liberia. Method: A total of 85 professors of the different majors have participated in this study and under a non-probabilistic sampling. A structured and closed questionnaire containing few questions was sent by e-mail utilizing the mail institutional platform and cellphones by the common digital platform WhatsApp. Results: The improvement of the pedagogic practice has a closed relationship with the good access to technologies and internet connection, which
\end{abstract}


has been considered a clear limitation for academics to communicate with students in an easy, better and effective way. Discussion: The academy needs to have the clarity in relation to the conditions that students face daily in order to study utilizing distant education spaces which in many of the cases do not have access to the technological tools and connectivity, most of that due to their bad social and economic conditions.

Keywords: teaching profession; online learning; education; learning; COVID-19.

\section{Introducción}

Luego de que la Organización Mundial de la Salud declarara el 11 de marzo de 2020 la situación de emergencia de salud pública por la enfermedad infecciosa denominada la COVID-19 en el ámbito internacional, muchos países ya habían previsto medidas para mitigar la propagación del virus causante de la misma, SARS-CoV-2. En Costa Rica, una de las medidas que se impuso para enfrentar la crisis del coronavirus fue la de cerrar, a partir de la segunda mitad del mes de marzo, los centros de educación (guarderías, escuelas, colegios), así como los centros de educación superior.

La enfermedad por coronavirus cambió de manera inesperada y radical la forma en que se imparte la educación, actualmente, el salón de clases y el hogar, son el mismo lugar. Según la Organización de las Naciones Unidas para la Educación, la Ciencia y la Cultura (UNESCOb), al 21 de mayo del 2020, existen más de 1,213,390,181 estudiantes que se han visto afectados por las restricciones debido a la pandemia en Costa Rica.

En marzo del 2020, la Universidad Nacional cerró sus puertas a las lecciones presenciales y de inmediato inició el proceso para que el personal docente diseñara los llamados "planes de contingencias", mediante los cuales se planificó el desarrollo de los cursos en la modalidad de presencialidad remota, con el objetivo de no interrumpir el ciclo académico. Luego del aislamiento temporal y brindar la continuidad a la educación, las instituciones universitarias han puesto la mirada en los beneficios de la educación virtual o educación con apoyo de herramientas tecnológicas. (Quintana, 2020).

Durante este proceso de adaptación cada docente asumió el reto de reinventarse rápidamente y empezar a trabajar de otra forma, diseñando actividades de aprendizaje y evaluativas en la presencialidad remota. En ese sentido, López (2017) advierte que la utilización de las TIC como herramientas de apoyo en la docencia deberían ser a partir de un modelo constructivista:

En la actualidad existen diversas herramientas digitales o ambientes interactivos de funcionamiento en Internet que permiten implementar actividades de aprendizaje bajo un modelo constructivista, con las cuales los estudiantes pueden construir su propio conocimiento a través de la elaboración de productos digitales (videos, animaciones, modelos, etc.) o la generación de estrategias para resolver desafíos o problemas a través de ambientes lúdicos. (p. 98)

El cierre de las instituciones educativas provoca desafíos significativos, ante un panorama que drásticamente acrecienta las brechas de aprendizaje entre la población. Los cambios vividos por la humanidad han transformado la forma de producir la vida, la sociedad y cultura, contrastando así una época de metamorfosis en las personas (Mejía, 2015).

Es en este contexto en el cual la educación virtual surge como la mejor opción para dar continuidad al proceso de enseñanza-aprendizaje, permitiendo a la población docente y estudiantil adaptarse a las disposiciones preventivas de aislamiento establecidas por la Presidencia de la República de Costa Rica y el Ministerio de Salud de Costa Rica (Cáceres, 2020). Es así como la educación en línea aparece en un contexto totalmente nuevo, como un proceso de enseñanza-aprendizaje, que cambia el currículum tradicional del pizarrón y salón de clases a una modalidad a distancia totalmente virtual.

El reto que se presenta es que por primera vez y en un corto período de tiempo y sin preparación previa debe de modificarse el proceso de enseñanza-aprendizaje y el profesorado, que impartía lecciones presenciales, se ve obligado a planificar, construir, medir y evaluar sus cursos de manera virtual desde sus casas directamente a los hogares de los alumnos, afrontando de esta manera tres 
grandes desafíos: actividades como enseñar online, demanda de un rediseño del sistema de evaluación y conocer las herramientas tecnológicas por implementar (García-Peñalvo, 2020d).

Desde el inicio de la pandemia una importante cantidad de docentes no se encontraban capacitados para establecer la virtualización total de sus clases, asumieron el reto de aprender en muy corto tiempo el uso de las herramientas TIC para desarrollar estrategias de mediación que les permitiera implementar sus clases, así como a tener la posibilidad de grabar y transmitir en vivo desde sus hogares, logrando comunicarse con sus educandos. El proceso de trasladar clases presenciales tradicionales a clases virtuales y la innovación que esto conlleva tuvo un efecto directo en el aumento de las horas laborales de los docentes, no solo en la preparación de material de mediación, sino también en el tiempo de atención a estudiantes en el transcurso de cada día. El utilizar herramientas TIC (Zoom, Microsoft Team, y otras) como apoyo a la docencia, ha permitido continuar el proceso de enseñanza por parte de cada docente, logrando esto sacar el máximo provecho a dichas herramientas. (García-Peñalvo, 2020e).

Durán (2020) afirma que:

Ante la actual pandemia, y debido a la situación de cuarentena que algunos países deben enfrentar, se dificulta tener actividades presenciales con el cuerpo estudiantil, por lo que todas aquellas metodologías que requieran de la interacción directa con los estudiantes ya no tienen total validez, y deben buscarse otras estrategias metodológicas virtuales para poder desarrollar el avance curricular asociado a los diferentes programas de actividad curricular. (p.12)

El segundo desafío ha sido la insuficiente conectividad y acceso a herramientas tecnológicas por parte del estudiantado, lo cual condiciona y restringe el acceso a los recursos digitales, como son las plataformas de videoconferencia utilizadas para la mediación del aprendizaje por parte del personal docente. La utilización de diferentes herramientas digitales facilita la comprensión de contenidos pedagógicos de estudiantes en clases virtuales (García-Peñalvo, 2020c).

Finalmente, un tercer desafío ha sido el rol que cada docente ha jugado en la motivación, apoyo psicosocial y emocional a sus estudiantes. El desconocimiento de la utilización de las TIC, afecta el desenvolvimiento de muchos estudiantes en la ejecución de sus tareas virtuales (Lozano-Díaz et al., 2020). Para la población estudiantil, el confinamiento ha afectado su proceso de enseñanzaaprendizaje en un área tan sensible como lo ha sido la imposibilidad de compartir con sus compañeros y docentes, en el centro de formación, socializando y permitiendo desarrollarse adecuadamente, y es aquí, en la virtualidad, donde el personal docente es llamado a propiciar un ambiente distendido, propicio para el aprendizaje, en el cual se interactúa y socializa (Vélez, 2020).

Ante estos desafíos, la Sede Regional Chorotega ha establecido acciones permanentes de apoyo en el uso de tecnologías de la información en la mediación pedagógica en los diferentes cursos impartidos, permitiendo que el personal docente tenga a disposición aplicaciones y recursos para potenciar la mediación en ambientes virtuales sincrónicos y asincrónicos. Tal y como lo precisan algunos autores múltiples variables de estrategias han efectuado los gobiernos para afrontar esta pandemia, en donde las medidas más frecuentes incluyen en campañas de información de salud, y aislamiento (Cobo-Rendón et al., 2020).

Sin duda alguna, ante las dificultades sobresalen las oportunidades, como es en la actualidad, que permite reflexionar sobre la carrera docente y la función de cada educador en el proceso de enseñanza-aprendizaje dentro de la innovación de herramientas de la tecnología de la información y la comunicación. Ante este panorama y con la intención de profundizar en los elementos que han influenciado al personal académico al pasar de la presencialidad a la virtualidad, es que se ha decidido realizar esta investigación en la Universidad Nacional de Costa Rica - Sede Regional Chorotega - Campus Liberia.

\section{Marco teórico}

Costa Rica reportó su primer caso de la COVID-19 en el mes de marzo, el cual coincidió con el inicio del año académico. La pandemia mundial sorprendió a los centros de educación superior. Con la interrup- 
ción de clases presenciales una gran cantidad de docentes de la Universidad Nacional Sede Regional Chorotega migraron sus cursos a plataformas en línea tales como Moodle, estableciéndose esta como la plataforma oficial de la universidad. De la misma forma se pusieron a disposición herramientas tecnológicas como Microsoft Teams, Google Meet, Office 365, Hangouts, entre otras.

Vera et al. (2014) explican este proceso:

Esta situación pone de manifiesto la existencia de necesidades de formación profesional y actualización docente en el uso de las TIC integradas al currículo. Por ello se hace necesario señalar como un problema la necesidad de diseñar medidas confiables y válidas, pero además proponer metodologías analíticas que nos proporcionen datos para la mejora continua en la formación del profesorado en el manejo de TIC en las universidades. (p.146)

Entre los múltiples retos que afronta la Universidad Nacional Sede Regional Chorotega, están el conocer cómo contender con los múltiples niveles de acceso que presentan los estudiantes a la tecnología, el acceso a internet y la falta de disponibilidad de equipo.

En ese sentido, Aguilar-Ródenas (2020) considera que:

El confinamiento por la COVID-19 nos ha dado la oportunidad de reflexionar sobre muchas cosas y, también, hemos comprobado todas aquellas cosas que no funcionan. El profesorado de todos los niveles educativos hemos tenido que adaptar las clases y la investigación para poder seguir trabajando con nuestro estudiantado. Un gran esfuerzo porque ha habido que improvisar, ha habido un problema de acceso a muchos recursos que se han tenido que suplir o que eran insuficientes o inexistentes en algunos casos, y en muchos hogares se ha añadido el problema de tener que cuidar de los hijos e hijas o de personas mayores o dependientes. (p.1)

En el caso de la Universidad Nacional, proveyeron a sus estudiantes con recursos para la conectividad, y la participación en los cursos, tales como tarjetas SIM a 3,000 estudiantes de todo el país, para su acceso a internet inalámbrico y 500 tablets en la Sede Regional Chorotega.

En lo que respecta a los efectos académicos, es importante destacar que pocos profesores tenían el entrenamiento o la experiencia necesaria para impartir sus lecciones en línea. En este contexto, la educación virtual se muestra como la opción que mejor puede adaptarse a las disposiciones preventivas de confinamiento establecidas por el gobierno de Costa Rica, puesto que ofrece múltiples posibilidades para el aprendizaje desde el hogar y así adquirir nuevos conocimientos en múltiples áreas, o continuar con el proceso aprendizaje, ante la amenaza de contagio.

Con relación a lo anterior, Cassany (2014) apunta lo siguiente;

Existen numerosas herramientas virtuales de las cuales profesores y alumnos hacen uso para llevar a cabo el proceso educativo. Todas y cada una de ellas presentan grandes ventajas y aportan numerosas cualidades para llevar a cabo el proceso de enseñanza centrado en el alumno, un proceso que se adapta a las exigencias que presenta tanto la sociedad. (p. 105)

Desde la aparición y propagación de la COVID-19 en nuestro país, los académicos universitarios se han abocado a la búsqueda de nuevas formas de afrontar el proceso enseñanza-aprendizaje para no interrumpir el ciclo lectivo. Cabe mencionar que algunos autores proponen mayor inversión en recursos humanos, y equipo tecnológico por parte de las universidades, y que deben ser capitalizados y orientados a aumentar su oferta educativa, con apoyo de herramientas tecnológicas, en tiempos de pandemia (Pérez-López et al., 2021).

De la misma forma se ha tenido que investigar y analizar el paso de lo presencial a lo virtual, dentro del marco de la legalidad estatutaria, y la realización del teletrabajo. Sin duda alguna este cambio de la modalidad presencial a la virtual para muchos académicos ha sido fácil, para otros docentes no tanto. En consecuencia, los efectos provocados por esta pandemia mundial han sido devastadores en todas las esferas académicas (García-Peñalvo, 2020a).

La oferta académica de las instituciones de educación superior en el país es extensa. Cada carrera propuesta presenta sus características y peculiaridades que hacen de este cambio de modalidad toda una novedad y desafío. Las universidades se han visto, de una forma muy rápida, en 
la necesidad de transformar radicalmente la manera tan arraigada de realizar su labor.

Para tal efecto Cassany (2014) establece:

Esta denominación genérica de "buenas prácticas" incluye elementos variados, que van desde tareas específicas (realizar búsquedas de información en la red) hasta rasgos bastante generales de un estilo docente (prescindir del libro de texto). Con todas las limitaciones que esto supone, nuestra intención consiste solo en agrupar diferentes características comunes que hemos hallado en los docentes [...]. (p.4)

Por más plazos que se concedan para establecer las ideas de cómo prosperar virtualmente, nos hallamos definitivamente en un contexto de inflexión del cual no hay vuelta atrás: la revolución digital. Actualmente, somos partícipes de un cambio en el acceso, almacenamiento y distribución de los recursos digitales, a decir de la digitalización electrónica de la información y comunicación (Mezarina et al., 2014).

La COVID-19 ha provocado que muchos profesionales cambien radicalmente su rutina laboral, entre ellos, los profesores. En esta investigación, se estudia la adaptación a esta nueva circunstancia y el cambio de metodología tradicional presencial a una a distancia a un corto plazo.

\section{Método}

Dado que, en la Universidad Nacional Sede Regional Chorotega, Campus Liberia, no ha sido abordada la temática desarrollada en esta investigación, el estudio realizado es de tipo exploratorio. Así también, es de carácter descriptivo, porque pretende detallar el impacto sufrido por los académicos en dicho centro de formación universitaria, a partir del cambio metodológico del proceso de enseñanza-aprendizaje, de modalidad presencial a modalidad virtual como consecuencia de la COVID-19.

\section{Diseño}

El estudio se diseñó partiendo de un enfoque de investigación mixto, de carácter cuantitativo que utiliza la recolección de datos, con el fin de establecer pautas de comportamiento (Hernández et al., 2014).
$\mathrm{Su}$ enfoque cualitativo se diferencia sustancialmente con la investigación cuantitativa en que, la primera no se basa en números o mediciones (Kerlinger \& Lee, 2002).

\section{Participantes}

En el estudio participaron 85 personas Docentes de las diferentes carreras de la Sede Regional Chorotega de la Universidad Nacional, Costa Rica, bajo un muestreo no probabilístico dentro de un período (del primer ciclo del 2020), donde las clases se desarrollaron desde la modalidad de presencialidad remota. Se empleó un cuestionario estructurado y cerrado de escasas preguntas enviado por correos electrónicos institucionales y por la vía telefónica mediante la aplicación digital WhatsApp.

Se utilizó una técnica de muestreo no probabilística, usando como marco muestral el número de docentes proporcionado por la Dirección Académica del Campus en estudio. Esta muestra establece margen de error de $5 \%$ máximo, con un $98 \%$ de confianza.

Esta muestra se compone de un $48.2 \%$ de profesores y un 51.8 \% de profesoras; del total, 19 imparten cursos en la carrera de Administración de empresas; 14 en la carrera de Ingeniería Hidrológica, 11 en Ingeniería en Sistemas de la Información, 10 en Gestión Empresarial para el Turismo Sostenible, ocho personas imparten lecciones en la carrera de Pedagogía, nueve en la carrera de Enseñanza del Inglés, siete en la carrera de Comercio y Negocios Internacionales; y siete imparten lecciones de Humanidades. En lo que a la edad de la población docente se refiere, el $42.4 \%$ cuenta entre 31 y 40 años, seguido por un 32.9\% de académicos con edades que oscilan entre los 41 y los 50 años. Un $21.2 \%$ de quienes imparten lecciones en el Campus Liberia tienen más de 50 años y la menor cantidad $3.5 \%$ se encuentran en un rango entre los 20 y los 30 años.

\section{Instrumentos}

Para la recolección de datos se diseñó un instrumento ad hoc, el cual se aplicó en línea con la plataforma Google Forms, para ser autoadministrado por la muestra de académicos. Los cuestionarios fueron aplicados en el período comprendido entre el 20 mayo al 7 de junio del 2020. 


\section{Procedimientos}

El procedimiento para llevar a cabo la presente investigación se estableció en tres etapas: en la primera etapa se diseñó un formulario en línea usando la plataforma Google Forms que responde a la técnica de la encuesta. La segunda etapa consistió en el envío vía correo electrónico a la población meta del estudio de la invitación a responder el cuestionario.

Los participantes fueron contactados a través del correo electrónico institucional de la Universidad. Además, el cuestionario fue aplicado por medios electrónicos (online) y, antes de aplicarlos, los participantes dieron su consentimiento explícito de participación voluntaria y anónima.

\section{Análisis de datos}

El análisis de los datos muestra un conjunto de resultados, que van dando respuesta a su vez al objetivo del estudio (Lozano-Díaz et al., 2020). Hay que mencionar que en esta etapa quedan establecidos para los participantes los aspectos éticos del desarrollo del estudio, respetándose por un lado su anonimato en la confección misma del cuestionario y garantizando un consentimiento implícito cuando cada participante decide individualmente, responder en la plataforma electrónica.

La etapa final consistió en el análisis de los datos, así como la interpretación de cada una de las respuestas. La respuesta a las preguntas abiertas de la población meta de la investigación fueron poscodificadas y detalladas con el método de minería de datos basado en identificar y cuantificar mediante métricas de análisis de texto y lenguaje natural.

De igual forma, el análisis estadístico empleado es un análisis descriptivo, basado en frecuencias y porcentajes. Adicionalmente, las distintas experiencias y percepciones que la población académica ha enfrentado ante el reto de una adaptación repentina de las clases presenciales a modalidades virtuales, se conoció a través de dos preguntas en el cuestionario que permitían la respuesta abierta. Los resultados arrojan que, en el contexto del campus, el personal académico encontró varias barreras para implementar desde la virtualidad la metodología de didáctica universitaria, debido al desconocimiento de plataformas y herramientas de mediación.

\section{Resultados}

En las primeras semanas de la cuarentena, los académicos iniciaron una fase retadora de autoaprendizaje para el uso de la tecnología de la información y la comunicación como apoyo a la docencia, lo cual implicó no solo la búsqueda de herramientas sino también aprender a utilizarlas y adaptarlas a las particularidades de cada clase, sin perder de vista el objetivo de una educación de calidad en pro de la formación de individuos críticos y analíticos. Por otro lado, el equipo de gestión administrativa inicia el proceso de reconocer la existencia de condiciones adecuadas dentro del campus universitario, como son la conexión a internet, equipo tecnológico (aula virtual) entre otros, para el acceso de personal docente.

Como parte del proceso, el equipo de gestión de administración educativa solicita a los docentes una minuciosa planificación de la mediación académica la cual parece haber generado, según lo indican los mismos docentes, mucha ansiedad, debido a la carencia de las destrezas digitales necesarias para la docencia en línea. En lo que a los resultados de la encuesta se refiere se muestra que el desconocimiento en la mediación pedagógica virtual es claro, puesto que el $70 \%$ del personal académico manifiesta que esta es la primera vez que imparten lecciones en modalidad virtual.

Este resultado explica la percepción de algunos y algunas docentes de que las clases presenciales se transformaron únicamente en "tediosas sesiones de videoconferencia", sin ninguna adecuación pedagógica. Sin embargo, un $85 \%$ del profesorado, considera que se han esforzado por investigar, innovar y proponer a la población estudiantil un conjunto de actividades para volver más provechoso el aprendizaje. Por ende, se pueden establecer que los primeros hallazgos en la investigación, la cual presenta como objetivo establecer el impacto del cambio metodológico del proceso de enseñanza-aprendizaje, de modalidad presencial a modalidad virtual como consecuencia de la COVID-19, en los académicos de la Universidad Nacional de Costa Rica - Sede Regional Chorotega - Campus Liberia.

Tal y como lo menciona Contreras (2020);

La manera de educar se está transformando, 
los sistemas presenciales están transitando a sistemas mixtos en donde convive la enseñanza presencial, las plataformas educativas y el uso de las TIC, además se ha ampliado la matrícula mediante sistemas de aprendizaje netamente virtuales. La transformación de la educación es irreversible, tanto en el uso de los medios como en los modelos educativos empleados, haciéndose necesario transitar de modelos clásicos a modelos constructivistas que se ajusten de mejor manera a la nueva realidad y permitan a los alumnos construir su propio saber. (p. 9)

Es indudable que la transformación pedagógica ante la crisis de la COVID-19 tomó a la academia por sorpresa. El desconcierto se ha dado en mayor medida en docentes que nunca se habían acercado a herramientas pedagógicas ya disponibles desde hace mucho tiempo como el aula virtual, Zoom, Microsoft Teams, Google Meet y todas las plataformas para la implementación de la educación virtual. Tal y como lo mencionan algunos autores consultados, estamos en la época de las herramientas tecnológicas como Zoom, Google Meet, Teams y otros tantos sistemas de videoconferencia. (Moreno-Rodríguez, 2020).

De la misma forma, dentro de los hallazgos de la investigación, se puede mencionar que la totalidad del personal académico ha tenido la oportunidad de conectarse a la red inalámbrica (WiFi) de su hogar, para realizar sus actividades docentes en la modalidad virtual; sin embargo, un $28.5 \%$ del profesorado reporta haberse conec- tado también al internet con sus datos celulares pospago y $4.7 \%$ con datos celulares prepago. Así mismo un $3 \%$ del profesorado ha impartido sus lecciones a través del internet de la universidad. Respecto al tiempo dedicado por día a diseñar y ejecutar sus clases virtuales, se observa en la figura 1 lo siguiente: el 44\% del personal académico dedica entre cuatro y siete horas a esas labores, el 29\% del personal docente reporta de una a cuatro horas diarias al diseño y ejecución de lección, un $25 \%$ manifiesta que son más de siete horas diarias las que se dedican a diseñar y ejecutar las clases, mientras que dos personas indican dedicar hasta 10 horas al día en estas actividades. Figura 1.

Estos resultados deben de analizarse considerando que un $83 \%$ del profesorado asegura estar dedicando mucho más tiempo para planificar y ejecutar sus clases en modalidad virtual cuando se compara con las clases presenciales. Ha sido evidente que los académicos con poco conocimiento en el uso de las herramientas tecnológicas han tenido que realizar un esfuerzo individual enorme, puesto que la cuarentena les impide contar con la ayuda de esos compañeros que solucionan los problemas informáticos en el marco de la institución universitaria. Este esfuerzo individual ha sido acompañado, por el apoyo de compañeros y compañeras universitarios tanto de la misma universidad como de otros centros de docencia superior, así como con la ayuda de hijos e hijas jóvenes de los docentes que participan en la búsqueda de recursos digitales.

\section{Figura 1}

Distribución de la muestra según variables sociodemográficas: género y deporte

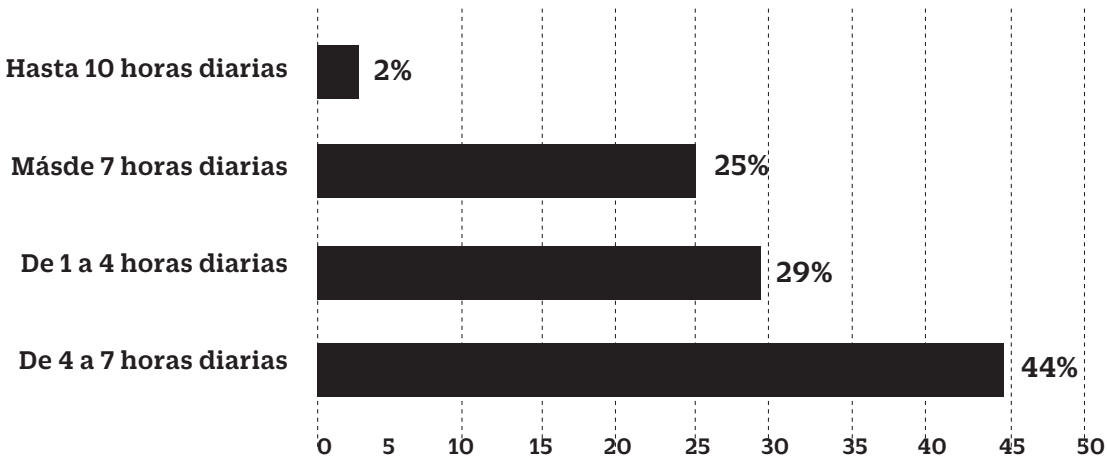


Este dato llama la atención, dado que el 70\% del personal académico menciona tener "suficiente dominio de las herramientas y estrategias de la enseñanza virtual" y solo un $30 \%$ considera que tiene poco dominio en el uso de herramientas y estrategias. Por lo que, es en otras aristas de las clases virtuales en las que se encuentra la explicación al tiempo adicional empleado. Las razones aducidas por el personal docente para estas labores suplementarias se relacionan, en términos generales, con el apoyo adicional a la población estudiantil, así, el personal académico considera que, para asegurarse la participación de los estudiantes al otro lado de la pantalla, deben de realizar actividades en cada tema, como tareas o foros, y esto luego se traduce en mucho trabajo por revisar para cada grupo. En este sentido, algunos académicos expresan opiniones como: "experiencia interesante pero agotadora".

El 70\% del personal académico considera que a los estudiantes les ha sido más difícil comprender algunos contenidos de la materia debido a la modalidad virtual, lo cual puede explicar la dedicación de más tiempo a la preparación de lecciones, ya que un $81 \%$ de la población académica siente que ha tenido que esforzarse más para apoyar a sus estudiantes en su aprendizaje en comparación con las clases presenciales. Aunado a lo anterior, un poco más de un tercio de los funcionarios y funcionarias encuestadas expresan sentirse presionados y abrumados por avanzar en los contenidos bajo esta modalidad virtual. Las circunstancias vividas con la emergencia sanitaria de la COVID-19 por el mundo entero se han manifestado de diversas maneras y el personal académico no es la excepción.

El personal académico menciona diversas situaciones particulares relacionadas con la pandemia que les ha perjudicado a ellos y a su núcleo familiar y han tenido una incidencia en su rendimiento como docentes. La salud mental se encuentra en el centro de lo apuntado, ya que se repite con regularidad la ansiedad, la presión y el estrés de estar constantemente viviendo en la "virtualidad" y además en convivencia permanente y confinamiento con miembros de la familia, quienes también requieren de atención, como hijos recibiendo clases virtuales. Se mencionan adicionalmente situaciones de orden físico como el dolor de cabe- za o de ojos por la exposición a las pantallas de las computadoras, o dolores de espalda por la cantidad de horas de estar sentados, para revisar, preparar, atender estudiantes y dar lecciones.

De igual manera se apunta que las horas trabajadas adicionales pueden deberse a una indebida organización del tiempo. A pesar de que siete de 10 docentes dicen tener un dominio suficiente en el uso de las herramientas y plataformas existentes, al mismo tiempo expresan la falta de conocimiento en el uso de las tecnologías existentes y una demanda por consejos y capacitaciones para el uso de las herramientas. Sin embargo, son pocos los que mencionan haber utilizado las plataformas de aprendizaje generadas por la universidad.

El porcentaje de uso de diferentes plataformas de mediación pedagógica utilizadas por el personal docente para impartir clases en la modalidad virtual se muestran en la figura 2. No es sorprendente que la plataforma institucional sea la más utilizada por el personal académico. Un 96.5\% utiliza para su mediación pedagógica el aula virtual institucional, adicional a esto se señalan el WhatsApp y Zoom con un $89 \%$ y un $83 \%$ de uso respectivamente. Es interesante notar la mención del WhatsApp, considerada por los docentes como una plataforma de mediación, aunque sea una aplicación de mensajería instantánea que permite el envío y la recepción de mensajes mediante Internet.

Dado que el personal académico puede enviar imágenes, vídeos, y grabaciones de audio este tipo de comunicación es considerada como una forma de mediar con el estudiante. Figura 2.

En lo concerniente a estrategias de mediación pedagógicas implementadas para impartir clases en la modalidad virtual, la figura 3 muestra cómo en prácticamente un $90 \%$ de los casos el personal docente acudió a tareas individuales solicitadas al estudiantado para transmitir sus clases. La siguiente estrategia más utilizada fue la videoconferencia o videollamada en tiempo real o sincrónica, lo cual coincide con las herramientas y plataformas mencionadas para impartir clases. Por otro lado, el 80\% del personal docente menciona en un tercer lugar los trabajos grupales como estrategia de mediación. Posiblemente estos trabajos, tanto grupales como individuales se relacionan con las otras estrategias mencionadas como lo son: 
Figura 2

Plataformas de mediación pedagógica, utilizado para impartir clases en la modalidad virtual.

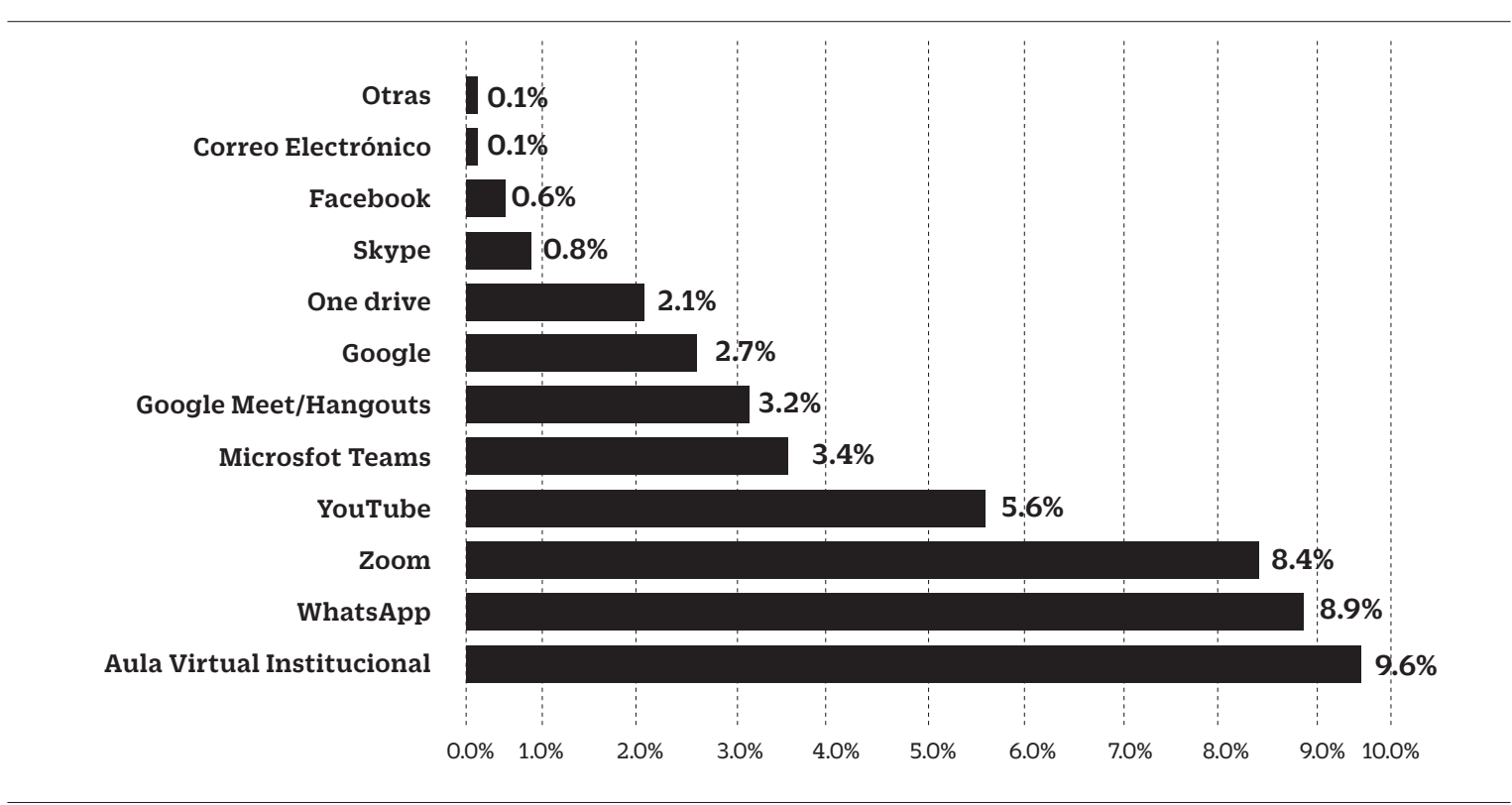

videos en la web (70.6\%), foros en línea (64.7\%), libros electrónicos (40\%) documentos escaneados (36.5\%) o cuestionarios en línea (50.6\%)

Dado el uso importante del aula virtual institucional, es altamente probable que las tareas y documentos se hayan entregado a través de las herramientas de archivos y etiquetas del aula, así como las entregas de tareas, las participaciones en foros, o los cuestionarios en línea. Es interesante destacar que un $40 \%$ del personal académico mencionó haber realizado videos pregrabados con las lecciones para ser luego mostradas a la población estudiantil. En este sentido es valioso anotar que el personal docente menciona reiteradamente que el hecho de tener lecciones grabadas ya sea por videos producidos por ellos mismos o por las lecciones grabadas en Meet, Zoom o similares, es una de las características positivas de la mediación virtual, ya que eso permite al estudiantado volver a escuchar y repasar las lecciones.

El 14\% del personal académico menciona otras estrategias de mediación, entre las cuales se incluyen: prácticas, Microsoft Power Point con voz grabada, documentos de elaboración personal, chats y grabaciones personales, artículos y notas de páginas web. Figura 3.

Los docentes universitarios hacen mención de varias acciones que se podrían implementar a fin de mejorar el proceso de virtualización de los cursos que imparten. Algunas actividades son: la necesidad de capacitación en entornos virtuales, tanto para el personal académico como para los estudiantes. Cabe aclarar que la Universidad Nacional ha realizado múltiples esfuerzos en este sentido desde la Vicerrectoría de Docencia y desde la Sede Regional Chorotega. Sin embargo, no es claro aún si estos esfuerzos han sido secundados por los docentes de manera individual, participando en ellos y utilizando la información que se ha puesto a su alcance.

En este sentido es interesante rescatar la mención de la necesidad de capacitación y consejos de mediación virtual que se adapten a cada especialidad. Las instituciones de educación superior están trabajando fuertemente en intentar mantener los métodos de aprendizaje utilizando orientaciones basadas en la virtualización (Hodges et al., 2020).

La mejora de la mediación pedagógica tiene estrecha relación con el acceso a la tecnología, tanto el equipo como el acceso al internet, y esta ha sido considerada una limitante para que el personal académico pueda comunicarse de manera fluida con la población estudiantil.

La superación del analfabetismo tecnológico puede ofrecer oportunidades de hacer cada vez una mejor universidad, es así que se hace necesa- 
Figura 3

Estrategias de mediación pedagógica, implementadas para impartir clases en la modalidad virtual.

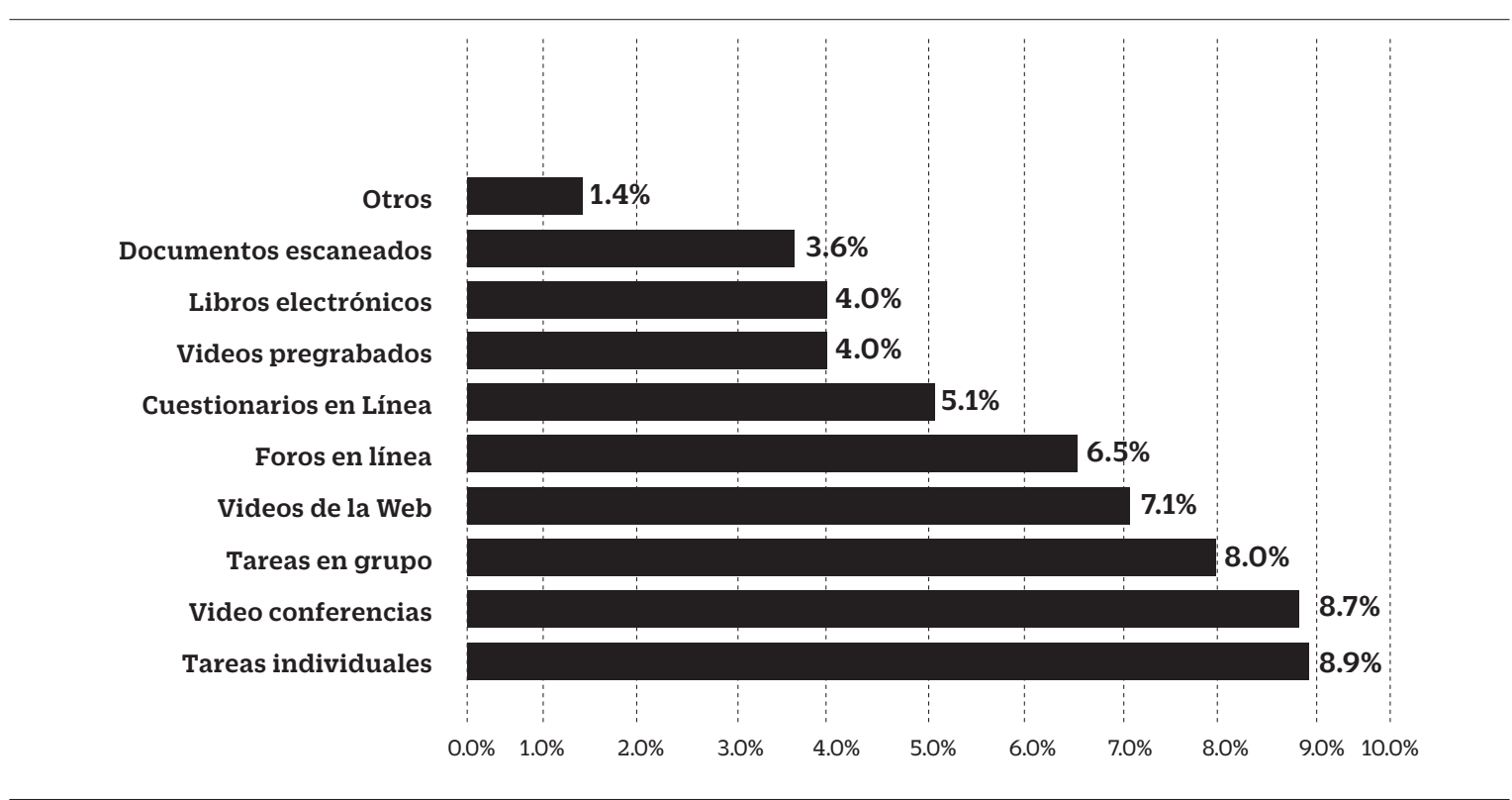

rio que desde la academia se incentive al personal docente a capacitarse en el uso de diversas plataformas sin limitarse al Aula Virtual y sus herramientas, sino también dominar otras herramientas y plataformas existentes que permitan no solo implementar clases virtuales sino también servir de apoyo cuando se imparten clases presenciales.

\section{Discusión}

Tal y como lo establece Álvarez et al. (2020), Sandoval (2020), Tejedor et al. (2020) los docentes han utilizado herramientas, a fin de fortificar los procesos de enseñanza aprendizaje, manifestando la necesidad de renovar conocimientos pedagógicos, didácticos y tecnológicos. De igual forma, los espacios como aulas virtuales son utilizados como opciones complementarias a la docencia continúa intercambiándolos ante las necesidades de beneficio de plataformas, tal y como lo describen autores como Crespo y Palaguachi (2020), De Luca (2020), Nuguer y Powell (2020) y Vergel (2020).

En este contexto, los resultados de otras investigaciones tales como las de Arancibia et al. (2020), Espinel (2020), Laro-González (2020), Medina et al. (2020) y Gutiérrez et al. (2020), referen- te a retos de la docencia en situaciones similares a una pandemia, establecen una apertura a nuevos conocimientos y una actitud positiva para integrar la formación presencial con ayuda de herramientas tecnológicas. Originando esto, innovaciones pedagógicas, como espacios para las actualizaciones complementarias y continuas en relación con las TIC.

Según lo menciona Molinero y Chávez-Morales (2019), para inicios de la pandemia el dispositivo más utiliza por los estudiantes en el salón de clases, es la computadora personal. Y las aplicaciones más utilizadas son Facebook, Drive, y YouTube.

Actualmente, en el contexto de la pandemia provocada por la COVID, las universidades presentan como reto proponer las condiciones al cuerpo de docentes, buscando la continuidad del proceso de enseñanza aprendizaje, y así evitar la interrupción del aprendizaje de los estudiantes, reduciendo distancias, procurando que el conocimiento no enfrente barreras. Hoy el docente universitario afronta un gran reto, como lo es la utilización de herramientas tecnológicas para la docencia universitaria, pasando de la presencialidad a la virtualidad.

Es importante establecer qué permutas no son fáciles de conseguir, esto debido a que la educación presencial tradicional ha cambiado por la 
pandemia provocada por la COVID-19, donde los docentes han tenido que echar mano a la utilización de herramientas TIC para prolongar sus métodos de enseñanza aprendizaje, beneficiando a educandos, que no ven truncado su proceso de aprendizaje.

Autores como Toribio (2016), hacen mención que son múltiples las experiencias de uso de TIC como apoyo a la docencia. De la misma forna Jordá-Borrell y López (2020) y Wenczenovicz (2020), mencionan que es ineludible prevalecer dificultades en la utilización de las herramientas TIC que consientan fortalecer el proceso de enseñanza aprendizaje.

El inicio de la virtualización de los procesos de enseñanza-aprendizaje no empieza con el contexto del COVID-19 (Llorens, 2020a). Con el inicio de la pandemia, causada por el virus SARS-CoV-2, las agendas de trabajo de los docentes se vieron interrumpidas, no por 40 días, si no por meses. Poco a poco se fueron llenando de nuevo, con acceso a conferencias y nuevos aprendizajes en línea a los que previamente no había acceso. Nos encontramos en un contexto, en el que se hace ineludible hacer un análisis sobre las maneras en las cuales se están transformando los procesos de educación en época del COVID-19 (Fardoun et al., 2020).

En este contexto, existe evidencia que se está ante una nueva realidad de la que se debe aprender y crear componentes y soluciones para transformar la virtualidad dentro del proceso educativo, por lo que en este horizonte el profesor debe construir metodologías alternativas para el desarrollo del material didáctico, en busca de que las gestiones para impartir sus clases sean claras, y se conserve un ambiente que favorezca la contribución del estudiantado. En relación con los elementos encontrados en esta investigación, los mismos concuerdan con lo establecido por Giannini, S. (2020), al establecer que los docentes no se encontraban preparados para afrontar esta transformación abrupta de lo presencial a lo virtual.

Existe un consenso de que como académicos se debe hacer todos los esfuerzos posibles por disminuir la brecha profunda que hay entre aquellos que cuentan con los recursos tecnológicos y aquellos que no. Aquellos que volverán a las aulas universitarias y aquellos que no. Y es en este contexto en un mundo entrelazado de dolor, ansiedad, miedo y preocupación, surge también un sentido de la oportunidad. La pandemia y sus consecuencias son la oportunidad para acelerar un proceso y manifestar aspectos en el mundo de la academia que tenían que suceder. Ha sido una invitación "forzada" de observar la realidad y buscar maneras diferentes de hacer docencia.

En la presente investigación se logró establecer que los docentes perciben que se requiere de mucho más trabajo en el proceso de enseñanza-aprendizaje bajo la modalidad virtual, esto debido a las múltiples labores que vienen a sumarse a las actividades docentes. Se incrementan las actividades académicas que demandan más tiempo frente al computador, más desgaste individual por la dificultad ante la explicación verbal de la materia de manera virtual, así como el aumento de revisión de trabajos debido a los cambios realizados en las evaluaciones, así como otras estrategias metodológicas. De la misma forma hay un aumento en el esfuerzo para realizar un replanteamiento de elementos académicos para alcanzar a toda la población estudiantil, dando seguimiento de forma grupal e individual, mediante el uso de herramientas tecnológicas como Meet, Zoom, Teams y WhatsApp, entre otras. Es indispensable precisar y filtrar los conceptos referentes a la educación en línea y la virtualidad, en donde la "educación de emergencia" que se está formalizando no siempre representa la modalidad (García-Peñalvo, 2020b). Los cambios en educación que ahora se están suscitando con apoyo de las herramientas tecnológicas brindan una serie de ventajas y beneficios, que hacen posible continuar con el proceso de enseñanza aprendizaje (García-Peñalvo, 2020f).

Los resultados obtenidos no son ajenos a otras investigaciones en las cuales se evidencia la novedad que estos procesos significaron tanto para la institución en general, como para los académicos en particular, y es en este aspecto que se debe dar una metamorfosis en los procesos virtuales, teniendo claro que: "la evaluación se une a la falta de experiencia institucional en este ámbito, pues no se han practicado ni probado estos procesos de forma no presencial" (García-Peñalvo et al., 2020, p. 12). Si bien denotan un desafío, existieron 
historias de éxito global de innovación en la elaboración de los profesionales actuales y futuros (Ferdig et al., 2020). Se está viviendo un momento sin precedentes en la historia de la educación superior en donde la utilización de herramientas tecnológicas como Zoom y Microsoft Teams, entre otras, brindan gran cantidad de beneficios y ventajas (García-Peñalvo et al., 2020). Con el aislamiento en nuestras casas, la comunidad universitaria se ha tenido que adecuar de emergencia a un modelo de docencia no presencial, lo cual ha favorecido y beneficiado al proceso de enseñanza aprendizaje (Llorens, 2020b).

Enseñar en los contextos de incertidumbre que produce la COVID -19 requiere creatividad y flexibilidad para permitir al estudiantado desarrollar nuevas habilidades y destrezas (Bosquez et al., 2018). Así como integrar nuevos conocimientos y aprendizajes, provenientes de los cursos en un ambiente virtual o en línea; esto con el objetivo de salir de esta situación con mejores herramientas y siendo mejores seres humanos (Ramírez et al., 2020). Los docentes se enfrentan a la formidable tarea, de contribuir en el proceso de enseñanza-aprendizaje y a la vez en la edificación de una realidad diferente, que permita otro tipo de educación. (Area-Moreira et al., 2018).

Así mismo, los docentes encuestados manifiestan que es preciso que se favorezcan capacitaciones, con el objetivo de adquirir estrategias donde los aprendizajes sean provocadores, motivantes y contextualizados y en los cuales se tome en cuenta el ritmo particular de cada participante.

Los resultados de esta investigación dejan claro una realidad, en la cual los docentes requieren una formación constante en lo que a la utilización de herramientas tecnologías como apoyo a la docencia se refiere, tanto para ser utilizadas en las clases presenciales como en la modalidad virtual. Y en eso llegó la pandemia... y los docentes fueron des-aulados y, en distinta medida, virtualizados (Fernández, 2020). La docencia online consiente un mayor control de la interacción de los educandos, condición que proporciona incluir esta participación en el contexto de evaluación continua de las disciplinas (Abella et al., 2020).

El sistema educativo no está realmente preparado para la educación a distancia, tal y como lo definen, Baptista-Lucio et al. (2020) los alumnos tampoco tienen la suficiente autonomía y responsabilidad en su propio aprendizaje. Los docentes se mueven en un margen acotado en la elección de contenidos. Durante la pandemia, al menos, los docentes han hecho lo que se puede, como una cuestión de supervivencia. Así como la pandemia transformó la higiene de la interacción social, también deberá generar cambios en la forma de enseñar y aprender de todas las figuras educativas.

En este marco se establece también un nuevo rol de la población estudiantil, razón de ser de la universidad y agente meta del entorno virtual. Sin duda alguna este asume su rol protagónico en el cual se le demanda la disposición para aprender, y desarrollar capacidades para el trabajo colaborativo y el autoaprendizaje desde la autonomía, de su hogar. La utilización de la tecnología ha tenido un impacto incuestionable en el proceso de enseñanza aprendizaje, donde la utilización de la misma ha presentado múltiples beneficios (Figueroa, et al., 2021).

Es por estas razones que es apremiante una restructuración del rol docente universitario. La crisis sanitaria y sus consecuencias han dado la oportunidad de generar un cambio en el cual para transformar lo presencial en virtual los docentes deban actualizarse en la utilización de herramientas tecnologías como apoyo a su práctica docente, se debe apuntar a una mejora en la capacitación relacionada con la ejecución de cursos a través de plataformas digitales, en métodos de evaluaciones digitales y en un cambio en los tiempos requeridos por los estudiantes en el proceso de enseñanza aprendizaje.

En síntesis, la función del docente en los procesos de aprendizaje virtual es reconocer a las realidades de los educandos, siempre tratando de lograr las metas educativas con un rol creativo, activo y afectuoso en respuesta a la comunidad estudiantil que convergen en las lecciones de forma virtual. Es así que la transformación del rol docente demanda de un proceso en el que coincidan tanto la capacitación como la disponibilidad de las herramientas tecnológicas, con el fin de lograr una organización que corresponda al contexto actual de virtualización de las lecciones. En un marco general, la mayor parte de universi- 
dades se halla en escenarios similares y enfrentan la situación mediante medidas heterogéneas dependiendo del caso determinado de cada nación (Grande de Prado et al., 2021). Lo indudable después de la pandemia provocada por el COVID-19 es que la educación universitaria como se conocía ya no existe más (Suárez, 2020).

En este marco, el personal docente debe de realizar transformaciones en el proceso de aprendizaje en el que la mediación pedagógica con presencialidad remota no sea una réplica de la clase presencial, y en el que se dé una coherencia pedagógica, entre los objetivos de aprendizaje, las actividades de mediación pedagógica, la evaluación y retroalimentación. El docente universitario conoce y aplica herramientas TIC, que facilitan el proceso de enseñanza aprendizaje (Navarro, 2020).

Esto propicia la interacción y la socialización, construir conocimientos y que el educando no solo sea un actor pasivo. Queda como tarea a los equipos de gestión académica el convocar a un proceso mediante el cual se trabaje no solo para la planificación de los próximos ciclos lectivos. La articulación de las herramientas TIC en el contexto virtual de las clases generada por los educadores, ha dado un nuevo impulso a los procesos pedagógicos (Sandoval, 2020).

En el futuro inmediato, se deben aprovechar las ventajas tecnológicas para impulsar una educación innovadora y colaborativa, en la cual el personal académico y la población estudiantil logren apropiarse de las herramientas tecnológicas: esto implementando una estrategia de actualización y capacitación constante del personal académico, en la utilización de recursos para el apoyo en la planificación y desarrollo de cursos en presencialidad remota. Sin duda alguna, la pandemia provocada por la COVID-19 ha impactado de forma perniciosa en los docentes universitarios en todo el mundo (Vidal et al., 2021).

El objetivo de esta investigación fue establecer el impacto del cambio metodológico del proceso de enseñanza-aprendizaje, de modalidad presencial a modalidad virtual como consecuencia de la COVID-19, en los académicos de la Universidad Nacional de Costa Rica - Sede Regional Chorotega - Campus Liberia. Del cual se desprenden algunas interrogantes;
¿Qué lecciones se ha aprendido de todo esto? Y aún se tiene pocas respuestas, han sido días difíciles. Ser académicos en la Universidad Nacional ha sido un soporte, no solamente económicamente sino también de manera espiritual. ¿Este sostén se está transmitiendo a los estudiantes, hoy fuera de las aulas? El campus universitario es un lugar de encuentro, pero ¿ahora? la Universidad se ha convertido en aquello que "gusta menos": no se tiene recreos, almuerzos en conjunto, ya los estudiantes no ven a sus amigos, los docentes no ven a sus colegas, en su lugar lo único que quedó son las tareas, las investigaciones, las exposiciones, las preparaciones sin fin de clases en presencialidad remota. Los estudiantes terminan abrumados y cansados de las guías de trabajo, las lecturas, los videos, los foros y tener que estar todo el día frente a la computadora y los académicos igualmente terminan sobrecargados y estresados.

Por último, aunque no menos importante, es que la academia tenga claridad sobre las condiciones de la población estudiantil, la cual no tiene las facilidades de acceso a las herramientas tecnológicas, conectividad y, especialmente, condiciones económicas, sociales y afectivas que les permita aprovechar sus máximas capacidades en los procesos de aprendizaje.

\section{Referencias}

Abella, V., Grande de Prado, M., García-Peñalvo, F. J., \& Corell, A. (2020). Guía de recomendaciones para la evaluación online en las Universidades Públicas de Castilla y León. Universidad de Burgos, Universidad de Salamanca y Universidad de Valladolid. https://bit. ly/2SqTtR2

Aguilar-Ródenas, C. (2020). Educación, género y coronavirus. Revista con la $A, 69$, 1-5. http://repositori.uji.es/ xmlui/handle/10234/188634

Álvarez, H., Arias, E., Bergamaschi, A., López, Á., Noli, A., Ortiz, M., Pérez, M., Rieble-Auborg, S., Rivera, M.C., Scannone, R., Vásquez, M., \& Viteri, A. (2020). La educación en tiempos del coronavirus: Los sistemas educativos de América Latina y el Caribe ante COVID-19. Banco Interamericano de desarrollo. https://doi.org/10.18235/0002337

Arancibia, M. L., Cabero, J., \& Marín, V. (2020). Creencias sobre la enseñanza y uso de las tecnologías de la 
información y la comunicación (TIC) en docentes de educación superior. Formación Universitaria, 13(3), 89-100. https://doi.org/10.4067/S071850062020000300089

Area-Moreira, M., San-Nicolás-Santos, M. B., \& Sanabria, A. L. (2018). Las aulas virtuales en la docencia de una universidad presencial: la visión del alumnado. Revista Iberoamericana de Educación a Distancia, 21(2), 179-198.

Baptista-Lucio, P., Almazán, A., Loeza, C. A., López-Alcaraz, V. A., \& Cárdenas-Domínguez, J.L. (2020). Encuesta Nacional a Docentes ante el COVID-19. Retos para la educación a distancia [Especial Issue]. Revista Latinoamericana de Estudios Educativos, 50, 41-88. https://doi.org/10.48102/rlee.2020.50.ESPECIAL.96

Bosquez, V., Sanz, C., Baldassarri, S., Ribadeneira, E., Valencia, G., Barragán, R., Camacho-Castillo, A., Shauri-Romero, J., \& Camacho-Castillo L. (2018). La Computación Afectiva: emociones, tecnologías y su relación con la educación virtual. Revista de Investigación Talentos, 5(1), 94-103. https://talentos.ueb. edu.ec/index.php/talentos/article/download/35/49

Cáceres, K. F. (2020). Educación virtual: Creando espacios afectivos, de convivencia y aprendizaje en tiempos de COVID-19. CienciAmérica, 9(2), 38-44.

Cassany, D. (2014). Cinco buenas prácticas de enseñanza con internet. Lenguaje y textos, Revista de la Sociedad Española de Didáctica de la Lengua y la Literatura, 39, 39-47. https://www.researchgate.net/ profile/Daniel-Cassany/publication/277015706_ Cinco_buenas_practicas_de_ensenanza_con_internet/links/555ef72a08ae6f4dcc8dfa20/Cinco-buenas-practicas-de-ensenanza-con-internet.pdf

Cobo-Rendón, R., Vega, A., \& García, D. (2020). Consideraciones institucionales sobre la Salud Mental en estudiantes universitarios durante la pandemia de Covid-19. CienciAmérica, 9(2), 277-284.

Contreras, N. M. M. (2020). El reto de la COVID-19, para la Educación en México. Revista Buen Gobierno, 29. https://doi.org/10.35247/buengob_29_06

Crespo, M. C., \& Palaguachi, M. C. (2020). Educación con Tecnología en una Pandemia: Breve Análisis. Revista Scientific, 5(17), 292-310. https://doi.org/10.29394/ Scientific.issn.2542-2987.2020.5.17.16.292-310

De Luca, M. P. (2020). Las aulas virtuales en la formación docente como estrategia de continuidad pedagógica en tiempos de pandemia. Usos y paradojas. Análisis Carolina. https://doi.org/10.33960/AC_33.2020

Durán, G. (2020). Educación en odontología para las asignaturas de simulación preclínica en tiempos de Pandemia por COVID-19. ODOVTOS International Journal of Dental Sciences, 22(2), 11-13. https://www. scielo.sa.cr/pdf/odovtos/v22n2/2215-3411-odovtos-22-02-10.pdf

Espinel, E. E. (2020). La tecnología en el aprendizaje del estudiantado de la Facultad de Ciencias Químicas, Universidad Central del Ecuador. Actualidades In- vestigativas En Educación, 20(2), 1-37. https://doi. org/10.15517/aie.v20i2.41653

Fardoun, H.M., González, C.S., Collazos, C., \& Yousef, M. (2020). Estudio exploratorio en Iberoamérica sobre procesos de enseñanza-aprendizaje y propuesta de evaluación en tiempos de pandemia. Education in the Knowledge Society, 21, 17. http://repositorio. grial.eu/handle/grial/2091

Ferdig, R. E., Baumgartner, E., Hartshorne, R., Kaplan-Rakowski, R., \& Mouza, C. (Eds.). (2020). Teaching, Technology, and Teacher Education During the COVID-19 Pandemic: Stories from the Field. Association for the Advancement of Computing in Education (AACE). https://bit.ly/2N7NT2L

Fernández, M. (2020). Una pandemia imprevisible ha traído la brecha previsible. https://bit.ly/2VT3kzU

Figueroa, C. G., Catuto, M. J., \& Salazar, H. (2021). El uso de las herramientas tecnológicas: un aporte al fortalecimiento de los aprendizajes. Revista Mapa, 5(23).

García-Peñalvo, F. J. (2020a). Evaluación online del aprendizaje: Reflexiones en tiempos de la COVID-19 [Webinar]. Grupo GRIAL. https://doi.org/10.5281/zenodo.3921801

García-Peñalvo, F. J. (2020b). Evaluación online: la tormenta perfecta. https://bit.ly/2yO3K39

García-Peñalvo, F. J. (2020c). El sistema universitario ante la COVID-19: Corto, medio y largo plazo. Universídad. https://bit.ly/2YPUeXU

García-Peñalvo, F. J. (2020d). Evaluación del aprendizaje en entornos virtuales y remotos[Webinar]. Grupo GRIAL. https://zenodo.org/record/3905200\#. YWpROBrMJPZ

García-Peñalvo, F. J. (2020e). Evaluación online durante la pandemia de la COVID-19. Caso de las universidades públicas de Castilla y León [Webinar]. Grupo GRIAL. https://zenodo.org/record/3874882\#. YWpW8xrMJPY

García-Peñalvo, F. J. (2020f). Modelo de referencia para la enseñanza no presencial en universidades presenciales. Campus Virtuales, 9(1), 41-56.

García-Peñalvo, F. J., Corell, A., Abella, V., \& Grande de Prado, M. (2020). La evaluación online en la educación superior en tiempos de la COVID-19. Education in the Knowledge Society, 21. https://doi.org/10.14201/ eks.23013

Giannini, S. (2020). Reconstruir mejor: tras el COVID-19, la educación debe cambiar para responder a la crisis climática. Organización de las Naciones Unidas para la Cultura, las Ciencias y la Educación (UNESCO). https://es.unesco.org/news/reconstruir-mejor-covid-19-educacion-debe-cambiar-responder-crisis-climatica

Grande de Prado, M., García Peñalvo, F. J., Corell, A., \& Abella, V. (2021). Evaluación en Educación Superior durante la pandemia de la COVID-19. Campus Virtuales, 10(1), 49-58.

Gutiérrez, R., Virgilio, V., Moreno, N., \& Maruri, C. (2020). 
Herramientas pedagógicas innovadoras en el Recinto "Urania Montás", San Juan de la Maguana, República Dominicana. International Journal of New Education, 5, 100-115. https://doi.org/10.24310/ IJNE3.1.2020.8511

Hernández, R., Fernández-Collado, C., \& Baptista-Lucio, P. (2014). Metodología de la investigación (6 $6^{\mathrm{a}}$ ed.). Mc Graw Hill.

Hodges, C., Moore, S., Lockee, B., Trust, T., \& Bond, A. (2020). The difference between emergency remote teaching and online learning. Educause Review. https:// bit.ly/3boNzx7

Jordá-Borrell, R., \& López, J. (2020). Factores de crecimiento económico en los países en desarrollo: el papel de las TICs. Boletín de la Asociación de Geógrafos Españoles, 86. https://doi.org/10.21138/bage.2979

Kerlinger, F. N., \& Lee, H. B. (2002). Investigación del comportamiento ( $4^{\mathrm{a}}$ ed.). McGraw-Hill.

Laro-González, E. (2020). Innovar enseñando: la educación del futuro. Las TICs como factor motivador en la enseñanza. REJIE Revista Jurídica de Investigación e Innovación Educativa, 21, 11-23. https:// doi.org/10.24310/REJIE.2020.v0i21.7530

Llorens, F. (2020a). Re-diseño exprés de la docencia para una adaptación de emergencia a la no presencialidad [Webinar]. Universidad Central del Ecuador. https://bit.ly/305k9vo

Llorens, F. (2020b). Docencia de emergencia: cómo cambiar el motor en pleno vuelo. Universídad. https://bit. ly/3cpHVEV

López, L. R. (2017). Indagación en la relación aprendizaje-tecnologías digitales. Educación y Educadores, 20(1), 91-105. https://www.redalyc.org/jatsRepo/834/83449754005/html/index.html

Lozano-Díaz, A., Fernández-Prados, J. S., Figueredo, V., \& Martínez, A. M. (2020). Impactos del confinamiento por el COVID-19 entre universitarios: Satisfacción Vital, Resiliencia y Capital Social Online. RISE, International Journal of Sociology of Education, 9(1) 79-104. https://doi.org/10.17583/rise.2020.5925

Medina, H., Lagunes, A., \& Guerra, M. T. (2020). ¿Qué aportan las Tecnologías de la Información y Comunicación en la enseñanza de las ciencias? Revista Digital Universitaria, 21(3). https://doi.org/10.22201/codeic.16076079e.2020.v21n3.a9

Mejía, M. R. (2015). Reconfiguración del capitalismo globalizado y resistencias desde América Latina. Nómadas, 43, 149-165. https://dialnet.unirioja.es/descarga/articulo/5428005.pdf

Mezarina, C., Páez, H.A., Terán, O., \& Toscano-Miranda, R. (2014). Aplicación de las TIC en la Educación Superior como estrategia innovadora para el desarrollo de competencias digitales. Campus Virtuales, 3(1), 88-101. http://uajournals.com/ojs/index.php/campusvirtuales/article/view/52

Molinero, M.C., \& Chávez-Morales, U. (2019). Herramientas tecnológicas en el proceso de enseñanza- aprendi- zaje en estudiantes de educación superior. RIDE Revista Iberoamericana para la Investigación y El Desarrollo Educativo, 10(19). https://doi.org/10.23913/ ride.v10i19.494

Moreno-Rodríguez, R. (2020). Reflexiones en torno al impacto del covid-19 sobre la educación universitaria: aspectos a considerar acerca de los estudiantes con discapacidad. Revista Internacional de Educación para la Justicia Social, 9(3), 1-6.

Navarro, S. (2020). Tendencias en el uso de recursos y herramientas de la tecnología educativa en la educación universitaria ante la pandemia COVID-19. Revista Ciencia y Tecnología El Higo, 10(2), 111-122.

Nuguer, V., \& Powell, A. (2020). 2020 Latin American and Caribbean Macroeconomic Report: Policies to Fight the Pandemic. Inter-American Development Bank. https://doi.org/10.18235/0002284

Organización de las Naciones Unidas para la Cultura, las Ciencias y la Educación (UNESCO). (2020). ¿Cómo estás aprendiendo durante la pandemia de COVID-19? https://es.unesco.org/COVID19/educationresponse

Pérez-López, E., Vázquez, A., \& Cambero, S. (2021). Educación a distancia en tiempos de COVID-19: Análisis desde la perspectiva de los estudiantes universitarios. RIED. Revista Iberoamericana de Educación a Distancia, 24(1), 331-350.

Quintana, I.(2020). Covid-19 y cierre de universidades ¿preparados para una educación a distancia de calidad? Revista Internacional de Educación para la Justicia Social, 9(3), 1-11. https://revistas.uam.es/riejs/article/download/12232/12094/31133

Ramírez I., Jaliri, C., Méndez, B., \& Orlandini, I. (2020). Percepciones universitarias sobre la educación virtual. Red de docentes IB, 3(1), 1-6. https://www.aacademica. org/ivonne.fabiana.ramirez.martnez/11.pdf?view

Sandoval, C. H. (2020). La Educación en tiempo del covid-19 herramientas tic: el nuevo rol docente en el fortalecimiento del proceso enseñanza aprendizaje de las prácticas educativa innovadoras. Revista Tecnológica-Educativa Docentes 2.0, 9(2), 24-31. https://doi.org/10.37843/rted.v9i2.138

Suárez, N. (2020). Formación docente universitaria y crisis sanitaria COVID-19. CienciAmérica, 9(2), 109-114.

Tejedor, S., Cervi, L., Tusa, F., \& Parola, A. (2020). Educación en tiempos de pandemia: reflexiones de alumnos y profesores sobre la enseñanza virtual universitaria en España, Italia y Ecuador. Revista Latina, 78, 1940. https://doi.org/10.4185/RLCS-2020-1466

Toribio, J. (2016). Las TICs en las universidades del CNU. http://www.cnu.edu.ni/las-tics-en-las-universidades-del-cnu/

Vélez, R. M. (2020). Retos de las universidades latinoamericanas en la educación virtual. Revista Virtual Universidad Católica del Norte, 59, 1-3. https://revistavirtual.ucn.edu.co/index.php/RevistaUCN/article/download/1140/1531 
Vera, J.A., Torres, L.E., \& Martínez, E, E. (2014). Evaluación de las Competencias Básicas en TIC en docentes de educación superior en México. Pixel-Bit. Revista de Medios y Educación, 44,143-155. https://www.redalyc.org/pdf/368/36829340010.pdf

Vergel, M. (2020). Educación, Covid y TIC. Revista Boletín Redipe, 9(8), 18-23. https://doi.org/10.36260/rbr. v9i8.1037
Vidal, M. J., Barciela, M. C., \& Armenteros, I. (2021). Impacto de la COVID-19 en la Educación Superior. Educación Médica Superior, 35(1), e2851. http://www.ems.sld. cu/index.php/ems/article/view/2851/1143

Wenczenovicz, T. J. (2020). Ensino a distância, dificuldades presencias: perspectivas em tempos de COVID-19. Revista Ibero-Americana de Estudos Em Educação, 15(4), 1750-1768. https://doi.org/10.21723/riaee. v15i4.13761

RIDU / Revista Digital de Investigación en Docencia Universitaria / e-ISSN: 2223-2516

(c) Los autores. Este artículo es publicado por la Revista Digital de Investigación en Docencia Universitaria del Área de Institutional Research and Effectiveness de la Dirección de Aseguramiento de la Calidad, Universidad Peruana de Ciencias Aplicadas. . "Este es un artículo de acceso abierto, distribuido bajo los términos de la LicenciaCreativeCommons Atribución 4.0 Internacional (CC BY 4.0) (https://creativecommons.org/licenses/by/4.0/deed.es), que permite el uso, distribución y reproducción en cualquier medio, siempre que la obra original sea debidamente citada." 